\title{
DE LA ESCUELA AL LABORATORIO ARQUEOLÓGICO: UNA EXPERIENCIA DIDÁCTICA
}

\author{
Isabel Muntalt Sánchez, Barbara Mura, Apen Ruiz Martínez \\ y Laura Trelliso Carreño ${ }^{1}$
}

\section{Resumen:}

La arqueología es una disciplina con un gran potencial educativo y social. En este artículo presentamos la experiencia didáctica De la excavación arqueológica al Laboratorio organizada por el Laboratorio de Arqueología de la Universidad Pompeu Fabra. Esta actividad forma parte del programa EscoLab, una iniciativa del Ayuntamiento de Barcelona, que pretende acercar la ciencia a los escolares de secundaria. A partir de un taller basado en la dinámica hands-on los alumnos se ponen en la piel de un investigador y reproducen el proceso de análisis e interpretación de los materiales arqueológicos.

\section{Palabras clave:}

arqueología, didáctica, hands-on learning, educación secundaria, cultura ibérica.

\section{Abstract:}

Archaeology is a discipline with a strong educational and social potential. In this article we present the didactic experience From the archaeological excavation to laboratory organized by the Laboratory of Archaeology at the University Pompeu Fabra. This activity is immersed in the EscoLab program, an initiative of the City of Barcelona, which aims to bring science to secondary school. By means of an activity based on dynamic hands-on, students put themselves in the shoes of a researcher and replicate the process of analysis and interpretation of archaeological materials.

\section{Key words:}

archaeology, didactics, hands-on learning, secondary school, Iberian.

\section{Introducción}

La actividad que se describe en este artículo titulada "De la excavación arqueológica al Laboratorio" está siendo realizada desde hace tres años por los/ las investigadores/as del Laboratorio de Arqueología de la Universidad Pompeu

1 Laboratori d'Arqueologia de la Universitat Pompeu Fabra. Barcelona. 
Fabra de Barcelona con alumnos de primer ciclo de secundaria. Esta experiencia didáctica se inserta en EscoLab: de la escuela al laboratorio, una iniciativa del Programa divulgativo Barcelona Ciència que el Institut de Cultura y el Programa de Cultura Científica del Institut d'Educació del Ayuntamiento de Barcelona vienen desarrollando desde el año 2008. El objetivo principal de EscoLab consiste en propiciar a los centros escolares la oportunidad de conocer y entrar en contacto directo con los laboratorios de investigación — de universidades y empresas - y con la/os científicas/os que trabajan en proyectos de investigación punteros en la ciudad de Barcelona y de su área metropolitana. El programa pretende acercar los alumnos de secundaria, ciclos formativos y bachillerato al ámbito de la investigación científica mediante la participación en actividades de muy diversa índole y formato: desde visitas guiadas por las instalaciones de los centros hasta talleres y ciclos de experimentación. La oferta de propuestas, más de cien y de amplio espectro, incluye temáticas relacionadas, entre otras, con las ciencias biomédicas y físicas, la tecnología y las ciencias sociales, como la arqueología.

La participación en estos talleres es gratuita y permite que los adolescentes se familiaricen con el día a día de la investigación científica: cómo funcionan los proyectos y particularmente el método de trabajo. En definitiva, se trata de que vean en qué y cómo trabajan las/los investigadoras/es para resolver las cuestiones que plantean sus proyectos. En los talleres experimentales, como el de arqueología que aquí presentamos, se invita al alumnado a que durante unas cuantas horas se ponga en la piel de un investigador y explore, experimente, cuestione e interprete un caso de estudio concreto mediante la aplicación de la metodología científica, tal y como haría un/a arqueólogo/a miembro de un proyecto de investigación. En este sentido, queremos contextualizar esta actividad dentro de un marco de referencia que se cuestiona las dimensiones sociales de la disciplina arqueológica, y en concreto la interacción entre educación y arqueología más allá de la transmisión de conocimiento histórico. ${ }^{2}$ Es decir, nuestra actividad no se hace desde la pedagogía ni desde el ámbito escolar sino desde un laboratorio de arqueología. Utilizamos los métodos de investigación arqueológica para acercar y revelar al alumnado la complejidad del proceso de investigación. Entendemos que desvelar las metodologías y los quehaceres de la ciencia desde un laboratorio universitario es nuestra forma de contribuir a la socialización del conocimiento arqueológico.

La arqueología suscita la fascinación y el interés de niños, jóvenes y adultos. A través de ella se abre un imaginario relacionado con la aventura y el enigma; una

2 P. GONZÁLEZ MARCÉN: Arqueología y enseñanza: la función social y educativa de los museos. Treballs d'Arqueologia (2002). pp. 1-8; B. SOLER MAYOR Y P. JARDÓN GINER: Descobrint la Prehistòria: una aplicació didàctica des de l'arqueologia experimental. Recerques del Museu d'Alcoi, 3 (1994) pp.149-152; G. RUIZ ZAPATERO: Presencia social de la arqueología y percepción pública del pasado. Museu de Prehistòria de València, 31 (2012). 
imagen que permite captar la atención y la curiosidad del público. A esta fascinación, a menudo impulsada por la literatura y los medios de comunicación, se suma el atractivo de la cultura material del pasado. Los objetos arqueológicos son testigos concretos de sociedades desaparecidas entorno a los cuales se construye no sólo una narración sino también una experiencia sensorial. Sin embargo, esta fascinación puede convertirse en un arma de doble filo, reduciendo la arqueología a una actividad de aventureros y al culto del objeto antiguo desvinculado del contexto socio-cultural del que procede. A través de un taller centrado en la metodología, enseñamos al alumnado cómo a partir del estudio de estos artefactos y del rastro de las actividades humanas -la cultura material- podemos reconstruir e interpretar la historia de nuestros antepasados evitando los estereotipos y los sesgos sexistas.

En lo educativo, todo elemento material y tangible ofrece un gran potencial didáctico. Los objetos, per se, permiten un aprendizaje activo dado que se pueden observar, manipular, comparar, ordenar $y$, ante todo, se les pueden interrogar. $^{3}$ Aprovechando dicho potencial y la curiosidad de los y las adolescentes, animamos al alumnado a interrogar la cultura material y a plantear una serie de hipótesis y preguntas a partir de la información recopilada. Se trata de un taller en el que se prioriza el aprendizaje crítico por encima del entretenimiento y de la exposición pasiva a una narración histórica. En este sentido, concordamos con Santacana cuando afirma que si la arqueología se reduce sólo a un elemento de entretenimiento y de ocio cultural, entonces sí que esta ciencia sería inútil. ${ }^{4}$

Así mismo, la arqueología hace uso de un método científico, sistemático y transparente en el proceso de documentación, descripción, análisis e interpretación de los artefactos. Explicar esta metodología puede dotar a los jóvenes de pautas concretas de razonamiento en las ciencias humanas y de una perspectiva crítica que pueden ser muy útil para su posterior formación. ${ }^{5}$ Además, su naturaleza interdisciplinar permite destacar la necesidad de desarrollar habilidades en el trabajo de grupo, en la comunicación, en el debate y la argumentación en público, rompiendo las barreras del individualismo y el estereotipo del científico solitario y alejado del mundo.

3 G. DURBIN, S. MORRIS Y S. WILKINSON: Learning from objects: a teacher's guide, English heritage, London:1990, pp. 4-6; J. SANTACANA I MESTRE: "L'arqueologia com a eina didàctica: problemas i utilitat". Cota zero, núm.15 (1999), pp. 63-73; A. BARDAVIO Y P. GONZÁLEZ MARCÉN: Objetos en el tiempo: Las fuentes materiales en la enseñanza de las Ciencias Sociales. ICE, Universitat de Barcelona, Editorial Horsori, Barcelona: 2003, pp. 83-102.

4 J. SANTACANA I MESTRE: "L'arqueologia com a eina didáctica: problemas i utilitat". Cota zero, núm.15 (1999), pp. 63.

5 J. SANTACANA I MESTRE: "L'arqueologia com a eina didáctica: problemas i utilitat". Cota zero, núm.15 (1999), pp. 63-73; J. BALUJA Y N. GONZÁLEZ MONFORT: "El patrimoni cultural: un objectiu didàctic?". Treballs d'Arqueologia, núm. 15 (2009), pp. 93-102; P. GONZÁLEZ MARCÉN: “La dimensión educativa en arqueología". I Congreso de prehistoria de Andalucía: la tutela del patrimonio histórico, Antequera (Septiembre 2010). 
Para finalizar, no podemos olvidar que dentro de la llamada socialización de la arqueología o de los usos sociales de esta disciplina ${ }^{6}$ se enmarcan también usos políticos que no siempre han estado al beneficio de la sociedad en su conjunto. Por ejemplo, es ampliamente sabido que las vinculaciones entre arqueología e imaginarios nacionales han colocado a la arqueología en una clara y problemática dimensión pública. ${ }^{7}$ En los últimos años se está repensando el carácter público y comunitario de esta disciplina, potenciando la participación activa de las comunidades y públicos diversos que se vuelven no sólo consumidores del discurso histórico, sino autores de narrativas alternativas que alteran órdenes sociales dominantes y discursos hegemónicos. Es en esta línea en la que se incluye nuestra actividad, mediante la cual pretendemos transformar la arqueología en una herramienta para que los estudiantes puedan reflexionar sobre el orden social en el que vivimos, sus orígenes, causas y consecuencias; favoreciendo la empatía histórica, el espíritu crítico y el pensamiento independiente.

\section{Propuesta didáctica}

\section{Objetivos de aprendizaje}

La formulación de los objetivos didácticos es un paso fundamental en todo proceso de enseñanza y aprendizaje, pues nos permite determinar aquellos conceptos, ideas, habilidades y aptitudes que los alumnos deberán adquirir, desarroIlar y asimilar. Se trata básicamente de responder a la pregunta ¿Qué queremos, realmente, que aprendan y en qué grado de profundidad? En la base del diseño y la elaboración de nuestra propuesta didáctica podemos destacar, de más general a concreto, los siguientes objetivos:

- Acercar la investigación a los jóvenes mediante el encuentro personal con nuestro equipo, proyecto e instalaciones de trabajo.

- Identificar el método de trabajo estructurado y sistemático que emplea la arqueología, como un ejemplo de ciencia social, que requiere de habilidades múltiples, espíritu crítico y del trabajo interdisciplinar.

6 R. GÓMEZ MARTÍNEZY J. NEGRE PÉREZ: "Arqueologia i acció social: De la inutilitat a la praxi, alguns exemples". Estrat crític, núm. 6 (2012), pp. 85-102; G. CARDONA: "Aprender con el patrimonio. Atención a la diversidad y patrimonio arqueológico en la educación secundaria obligatoria: estudio de casos en Cataluña". En I Congreso Internacional de Educación Patrimonial: formación y accesibilidad al patrimonio, Madrid (Octubre 2012); P. GONZÁLEZ MARCÉN: "La dimensión educativa en arqueología". I Congreso de prehistoria de Andalucía: la tutela del patrimonio histórico, Antequera (Septiembre 2010).

7 M. DÍAZ-ANDREU Y T. CHAMPION: Nationalism and Archaeology in Europe, UCL Press, London:1996; B. TRIGGER: A history of archaeological thought, Cambridge University Press, Cambridge: 2006 . 
- Comprender que las tareas de excavación en arqueología comportan la destrucción parcial o completa de los yacimientos y de su información; ello conlleva la necesidad de registrar con rigurosidad todos los datos.

- Valorar el patrimonio histórico y cultural como un espacio de memoria colectiva cuya herencia debe preservarse. Potenciar la conciencia histórica para que los jóvenes puedan comprender el pasado y valorar el presente individual y colectivo.

- Reconocer la cultura material como un instrumento de análisis social que nos ayuda a comprender la construcción social tanto de las sociedades del pasado como del presente.

\section{Estructura y temporización}

La actividad se desarrolla a lo largo de una mañana y consta de tres partes: un bloque teórico sobre la metodología arqueológica; una breve presentación de un proyecto arqueológico actual y por último, un taller práctico. En el apartado teórico se exponen, de manera sucinta, los principios básicos del método arqueológico, las técnicas y las diversas disciplinas que intervienen en la investigación. En la segunda parte, explicamos el proyecto arqueológico que llevamos a cabo desde el Laboratorio de Arqueología de la UPF. Este ejemplo concreto nos sirve para mostrar la aplicación de la metodología que han visto anteriormente así como las diferentes fases que componen todo proceso científico, desde la excavación y el trabajo en el laboratorio hasta la publicación y divulgación de la investigación. Por último, se desarrolla la parte práctica que es el núcleo de la actividad. Consiste en un caso de estudio ficticio que permite, mediante la dinámica hands-on, que los alumnos se pongan en la piel de un investigador y reproduzcan sus pasos en las tareas de análisis e interpretación de los materiales. El aprendizaje hands-on es un método educativo que implica directamente a los alumnos en la realización de una tarea con el objetivo de aprenderla. Esta dinámica didáctica, muy extendida en la actualidad, potencia elementos decisivos del proceso de aprendizaje como una actitud activa y participativa, y además, fomenta la experimentación en primera persona a través de la manipulación de los materiales (Fig.1).

\section{Caso de estudio y materiales}

La construcción de un caso de estudio nos permite, por un lado, contextualizar y dar sentido a la actividad a través de una narrativa ficticia que marque el punto de partida y el objetivo final de la actividad propuesta. Y por otro, ejemplificar el paso del trabajo de campo al del laboratorio que hemos explicado en la sesión teórica.

En el Vendrell, un agricultor ha encontrado en lo alto de una colina una estructura de piedra que probablemente sea un muro. La Agrupación de Amigos 
del Patrimonio Histórico del Vendrell ha pensado que se pueden tratar de unos restos con valor histórico y ha contactado con nuestro laboratorio para contextualizar los vestigios, ya que la gente de la zona recuerda que paseando por la zona habían encontrado en la superficie fragmentos de cerámica, probablemente de época antigua.

Nuestro laboratorio ha aceptado la colaboración pero primero de todo necesitamos el permiso legal de las instituciones competentes (en este caso, el Ayuntamiento y el Servicio de arqueología) para poder llevar a cabo la intervención. Una vez está la documentación en regla, es necesario planificar el proyecto.

En el terreno, hacemos una limpieza superficial para delimitar la extensión de la estructura y sus características. Dibujamos la planta y registramos los objetos encontrados en la superficie, sólo cerámica. Como necesitamos hacer un informe para determinar las características del yacimiento y su valor patrimonial, hemos decidido hacer tres sondeos de $2 m \times 2 m$ en tres espacios diferentes de la estructura para saber de qué se trata.

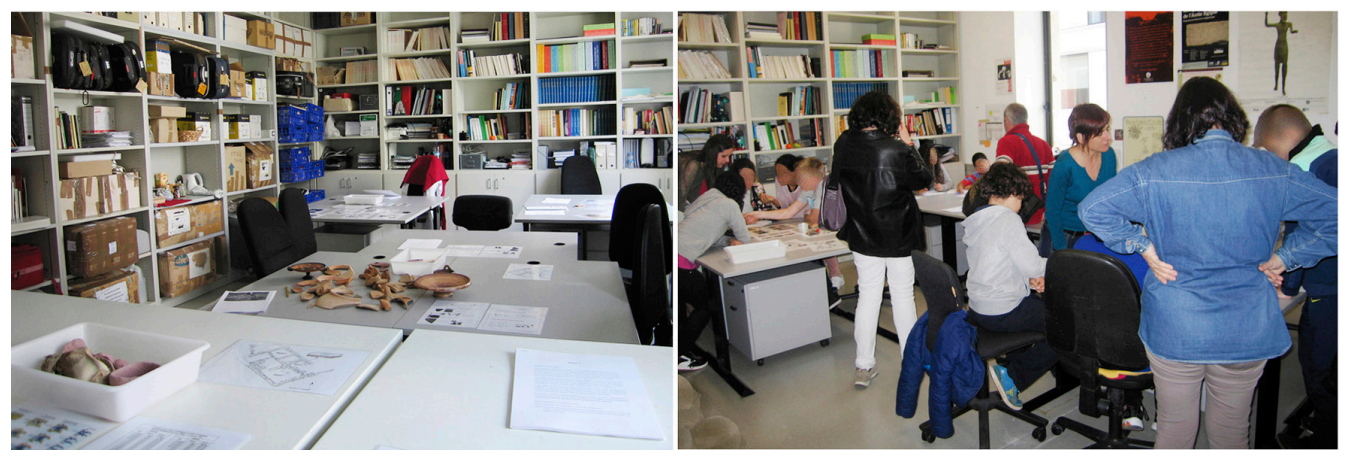

Fig. 1. Vista general del taller De la excavación arqueológica al laboratorio, antes y durante de la actividad. Marzo de 2015. Fotos: @ LARQ/UPF.

Con este caso de estudio ficticio proporcionamos a los alumnos la planta de una estructura que ha sido excavada rigurosamente. Ellos deberán analizar los restos materiales hallados en dicha estructura e interpretarlos. Ésta, de forma rectangular, corresponde a una unidad doméstica ibérica con enterramiento infantil. La elección de un caso ibérico responde esencialmente a dos elementos. Primero, que se trata de un ejemplo geográfica y culturalmente próximo a los alumnos y, segundo, que se contempla en el currículum escolar por lo que se trabajará también en el aula escolar.

A la hora de seleccionar y preparar los materiales del caso de estudio buscamos siempre ser lo más fiel posible al método y las herramientas que empleamos en el laboratorio. Los alumnos disponen de tres recursos diferentes: un investiga- 
dor que les dirige y ayuda en todo momento, diferentes registros documentales basados en nuestras propias fichas de trabajo (fotografías, base de datos, dibujos y planimetría) y una selección de materiales arqueológicos: cerámica, huesos humanos, restos de botánica y fauna etc. Estos materiales son distribuidos en cuatro mesas temáticas:

- Cronología: estratigrafía, escritura, tipología y seriación.

- Espacio 1: consumo/ cocina/ alimentación y ecosistema.

- Espacio 2: talleres: materias primeras y producción.

- Espacio 3: mundo funerario y perfil biológico.

\section{Dinámica de trabajo}

La propuesta didáctica está diseñada para unos 20 a 25 alumnos divididos en cuatro grupos correspondientes a cada una de las mesas que componen la actividad. Se pueden plantear dos opciones de trabajo. O bien todos los grupos rotan por cada una de las mesas para trabajar los materiales de forma íntegra y extraer sus propias conclusiones entorno a la estructura, o bien; a cada grupo se le es asignado uno de los espacios trabajando de este modo un único tipo de material. En este caso, cada grupo de alumnos deberá exponer los resultados a sus compañeros, y una vez obtenidos todos los datos, llegar a una conclusión conjuntamente. Sendas opciones favorecen el trabajo interdisciplinar, estimulan las dinámicas en grupo y trabajan la comunicación, el debate y la argumentación en público (Fig.2).

El objetivo es muy claro, los alumnos deberán identificar los usos de cada uno de los espacios y posteriormente, interpretar en conjunto la función de la estructura. Las pautas a seguir son muy concretas, deberán completar una serie de pasos que les llevarán de lo concreto a lo abstracto y de las diferentes partes al todo:

- Identificar, clasificar y cuantificar los restos.

- Realizar hipótesis sobre la posible función de los diferentes espacios.

- Adquirida la visión de conjunto proponer una interpretación de la estructura en sí.

- Y por último, efectuar una interpretación de los datos obtenidos. Es decir, a través, del análisis del espacio 1 (cocina) podrán reflexionar sobre temas de consumo y alimentación, ecosistema y medios de subsistencia. El espacio 2 (taller) ofrece la posibilidad a los alumnos de tratar aspectos relacionados con las materias primas y los sistemas de producción a diferentes escalas. Y el espacio 3 (enterramiento) permite debatir sobre las costumbres funerarias y el perfil demográfico de la población (edad, género etc.). 

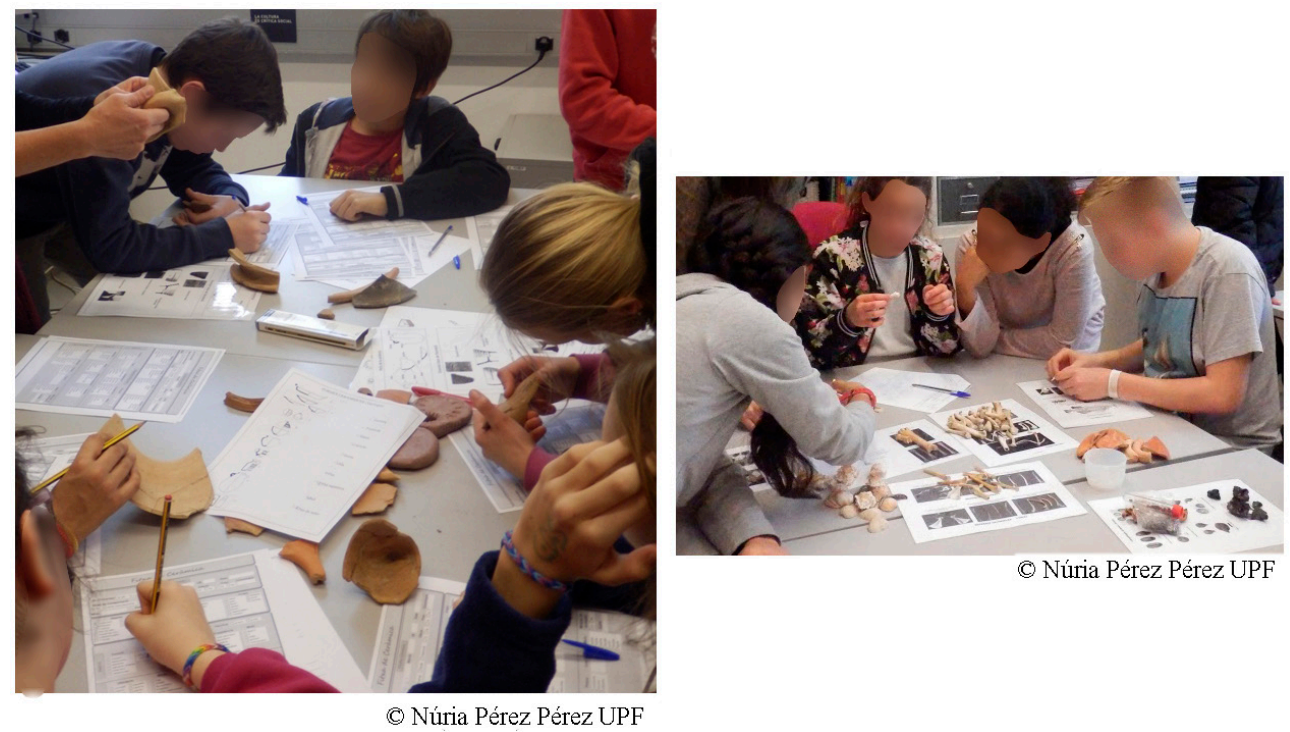

Fig. 2. Alumnos y alumnas de primero de Eso durante el taller práctico en las ediciones de 2014 y 2015. Fotos: (c) Núria Pérez Pérez. UPF.

\section{Reflexión y retos}

Después de las tres experiencias didácticas nuestra valoración es positiva y de aprendizaje mutuo. No sólo hemos acercado el mundo de la arqueología a alumnos de secundaria sino que los alumnos nos han aproximado a una realidad educativa que nos demanda repensar nuestra disciplina de manera diferente. Durante el proceso han surgido diversos retos relacionados con los dos agentes que interactúan en esta actividad: nuestro equipo y la escuela (profesorado y alumnos).

En primer lugar, nuestro equipo. A pesar de nuestros esfuerzos por dar continuidad a esta iniciativa, la proyección en el futuro es difícil. Todos sabemos de la poca estabilidad y la precariedad actual en el mundo de la investigación. Otros retos para el equipo han sido la creación del taller práctico y el proceso de preparación y adaptación de los materiales didácticos sin simplificar el contenido. Es decir, nos enfrentamos con un problema de transferencia de conocimientos y traducción de lenguajes especializados. En este caso por ejemplo, decidimos que nuestro objetivo primordial era ser lo más fiel posible al método y las herramientas que empleamos en el laboratorio, y por este motivo, tomamos como base los protocolos de registro que usamos en nuestras excavaciones sin adaptarlas a un contexto de estudiantes de escuela secundaria. En segundo lugar, la escuela y los alumnos. Es evidente que cada escuela es diferente respondiendo a un modelo educativo determinado y los escolares presentan perfiles socioculturales y económicos distintos, diferentes niveles de conocimiento y mecanismos de 
aprendizaje. Conseguir realizar una actividad que logre satisfacer las necesidades de todos ellos sin conocerlos previamente es una tarea bien difícil. Es por ello que ante toda esta complejidad decidimos enviar con anterioridad un breve formulario para saber a priori si los alumnos poseen una base de conocimientos previos sobre el tema, si han realizado actividades similares o han visitado alguna excavación. Así como, por qué los profesores han escogido esta actividad, cómo creen que se puede relacionar con el programa de estudios en Ciencias Sociales de la escuela y qué puede aportar al temario.

\section{Conclusiones}

El trabajo de divulgación del conocimiento arqueológico y la tarea de promoción de actividades didácticas dirigidas a escolares y familias ha sido llevado a cabo tradicionalmente por los museos e instituciones culturales. La iniciativa EscoLab ha proporcionado al Laboratorio de Arqueología la posibilidad de aportar nuestro grano de arena en este sentido. Nuestra actividad se enmarca en la línea de muchas otras pero con la innovación de abrir las puertas de un centro de investigación a los jóvenes.

Finalmente, queremos destacar dos aspectos. En primer lugar, el carácter social, público y comunitario de la arqueología exige un compromiso de divulgación. Por este motivo, es necesario que arqueólogos, docentes y didactas trabajen de forma conjunta para superar la desconexión que existe en la actualidad entre la investigación, el mundo educativo y nuestra sociedad. Y en segundo lugar, debemos utilizar y maximizar el potencial educativo y didáctico de nuestra disciplina. Gracias a su contenido práctico, a su carácter material y método, la arqueología tiene mucho que ofrecer a la diversidad de nuestra realidad escolar. Para ello, necesitamos interactuar y dialogar más con la escuela, con los profesores y alumnos, para saber cuáles son sus necesidades e intentar dar respuesta. Tras nuestra experiencia podemos concluir que esta actividad planteada como Handson learning para los estudiantes, al final se ha convertido en un learning by doing para nosotros.

\section{Agradecimientos}

Deseamos expresar nuestro más sincero agradecimiento a todas aquellas personas que con su ayuda han facilitado la realización de nuestra actividad dentro de Escolab. A Núria Pérez Pérez (UPF) por su constante apoyo, colaboración y estímulo. Al equipo del Institut de Cultura del Ayuntamiento de Barcelona por invitarnos al programa de Escolab. A las escuelas e institutos, alumnos y profesorado del Joan Salvat Papasseit y de la Escola Projecte por su participación en nues- 
tra actividad. A Francesc Gracia y David Garcia i Rubert (Catedrático y Profesor Asociado, respectivamente, del Departamento de Prehistoria, Historia Antigua y Arqueología de la Universidad de Barcelona por la préstamo temporal de material didáctico. Y, por último, a C. Baqué del MAC de Girona.

\section{Bibliografia}

J. BALUJA Y N. GONZÁLEZ MONFORT: "El patrimoni cultural: un objectiu didàctic?". Treballs d'Arqueologia, núm. 15 (2009), pp. 93-102.

A. BARDAVIO Y P. GONZÁLEZ MARCÉN: Objetos en el tiempo: Las fuentes materiales en la enseñanza de las Ciencias Sociales. ICE, Universitat de BarceIona, Editorial Horsori, Barcelona: 2003, pp. 83-102.

A. BARDAVIO, C. GATELL ARIMONT, P. GONZÁLEZ MARCÉN Y R. SOLSONA FONTOVA: "Una aproximació didáctica a l'arqueologia de la guerra civil: el cap de pont de Balaguer". Ebre, vol.38, núm.6 (2011), pp. 239-254.

A. BARDAVIO NOVI, P. GONZÁLEZ MARCÉN Y J. PIZARRO BARBERÀ: "Experimentació didáctica en arqueología. El projecte educatiu del camp d'aprenentatge de la noguera". En Experimentación en arqueología. Estudio y difusión del pasado. Sèrie Monogràfica del Mac, (Girona 2013), pp. 25-30.

G. CARDONA: "Aprender con el patrimonio. Atención a la diversidad y patrimonio arqueológico en la educación secundaria obligatoria: estudio de casos en Cataluña". En I Congreso Internacional de Educación Patrimonial: formación y accesibilidad al patrimonio, Madrid (Octubre 2012).

M. DÍAZ-ANDREU Y T. CHAMPION: Nationalism and Archaeology in Europe, UCL Press, London:1996.

G. DURBIN, S. MORRIS Y S. WILKINSON: Learning from objects: a teacher's guide, English heritage, London:1990, pp. 4-6.

J. GARCIA ROSSELLÓ, D. JAVALOYAS MOLINA, D. ALBERO SANTACREU, Y M. CALVO TRIAS: "El aprendizaje en grupo fuera del aula: los talleres didácticos de arqueología". III Congreso Internacional Univest, Girona (Junio 2011).

R. GÓMEZ MARTÍNEZ Y J. NEGRE PÉREZ: "Arqueologia i acció social: De la inutilitat a la praxi, alguns exemples". Estrat crític, núm. 6 (2012), pp. 85-102.

P. GONZÁLEZ MARCÉN: Arqueología y enseñanza: la función social y educativa de los museos. Treballs d'Arqueologia (2002). pp. 1-8.

P. GONZÁLEZ MARCÉN: "La dimensión educativa en arqueología". I Congreso de prehistoria de Andalucía: la tutela del patrimonio histórico, Antequera (Septiembre 2010). 
D. JAVALOYAS: "Aprendiendo a través de la basura. Una experiencia educativa en arqueología". Íber Didáctica de las Ciencias Sociales, Geografía e Historia, 75 (Octubre 2013), pp. 90-96

G. RUIZ ZAPATERO: Presencia social de la arqueología y percepción pública del pasado. Museu de Prehistòria de València, 31 (2012).

J. SANTACANA I MESTRE: "L'arqueologia com a eina didáctica: problemas i utilitat". Cota zero, núm.15 (1999), pp. 63-73.

B. SOLER MAYOR Y P. JARDÓN GINER: Descobrint la Prehistòria: una aplicació didàctica des de l'arqueologia experimental. Recerques del Museu d'Alcoi, 3 (1994) pp.149-152.

R. SKEATES, C. MCDAVID \& J. CARMAN: The Oxford Handbook of public Archaeology, Oxford University Press, Oxford: 2012.

B. TRIGGER: A history of archaeological thought, Cambridge University Press, Cambridge: 2006. 
